\title{
Review \\ Tumour-stroma interactions \\ Phenotypic and genetic alterations in mammary stroma: implications for tumour progression
}

\author{
Seth L Schor and Ana M Schor \\ Unit of Cell and Molecular Biology, The Dental School, University of Dundee, Dundee, Scotland
}

Correspondence: Seth L Schor, Unit of Cell and Molecular Biology, The Dental School, University of Dundee, Dundee DD1 4HR, Scotland. Tel: +01382 635 991; fax: +01382635 998; e-mail: s.l.schor@dundee.ac.uk

\begin{abstract}
In addition to the well documented role of cytokines in mediating tissue-level interactions, it is now clear that matrix macromolecules fulfil a complementary regulatory function. Data highlighted in the present review extend the repertoire of matrix signalling mechanisms, (1) introducing the concept of 'matrikines', these defined as proteinase-generated fragments of matrix macromolecules that display cryptic bioactivities not manifested by the native, full-length form of the molecule, and (2) indicating that a previously identified motogenic factor (migration stimulating factor [MSF]) produced by foetal and cancer patient fibroblasts is a genetically generated truncated isoform of fibronectin, which displays bioactivities cryptic in all previously identified fibronectin isoforms. These observations are discussed in the context of the contribution of a 'foetal-like' stroma to the progression of breast cancer.
\end{abstract}

Keywords: cancer progression, cell motility, epithelial-stromal interactions, fibronectin

\section{Introduction}

The pathogenesis of common human cancers is driven by the progressive accumulation of genetic lesions within the target epithelial cell population. Perturbations in the functionality of particular constellations of these oncogenes and tumour suppressor genes gradually confer the emerging population of premalignant and malignant cells with a proliferative advantage compared with their normal counterparts, as well as increasingly aberrant phenotypic characteristics, such as the capacity for local invasion and metastasis [1,2]. During the past few years, emphasis has gradually shifted away from essentially cataloguing these genetic lesions to functional studies that are concerned with their consequences on gene expression and with the intracellular pathways that are responsible for manifesting perturbed epithelial cell behaviour [3].
Although this focus on the target epithelial cell population is central to our understanding of cancer pathogenesis, it has long been our contention that such an exclusively 'epitheliocentric' view is too restricted and that regulatory signals that originate in surrounding tissues also make a clinically significant contribution to the kinetics of disease progression $[4,5]$. This view now sits comfortably within mainstream thought. It is supported by a number of contemporary reviews that concluded that stromal and epithelial cells exert reciprocal effects on the behaviours of each other, and that these essentially epigenetic, tissue-level interactions result in 'cell activation' and thereby contribute to tumour progression [6]. No rigorous criteria that define cell activation are currently available. It is generally accepted, however, that these collectively involve the re-expression of certain 'foetallike' phenotypic characteristics, including changes in cell

MSF = migration stimulating factor 
morphology and the secretion of various cytokines, 'oncofoetal' isoforms of matrix macromolecules and matrixdegrading enzymes. Acting together, these molecules orchestrate important tissue-level events, including directional cell migration (chemotaxis), matrix remodelling and the in-growth of new blood vessels (angiogenesis).

The present short review focuses on the role played by matrix macromolecules produced by activated cells (both stromal and epithelial) in the pathogenesis of breast cancer. Specific attention is given to the following: the manner by which these molecules co-operate with cytokines in the regulation of cell behaviour; the expression of relevant cryptic bioactivities by protease-generated fragments of matrix macromolecules; recent observations from our laboratory that indicate that similar cryptic bioactivities are also expressed by a recently cloned genetically truncated isoform of fibronectin; and the origins and clinical implications of stromal cell phenotypic diversity in breast tumours.

\section{Matrix involvement in the mediation of epithelial-stromal interactions}

Interactions between adjacent epithelial and stromal tissues play a key role in defining the spatial and temporal pattern of morphogenesis during embryonic/foetal development, and continue to contribute to the maintenance of normal cell function throughout adult life [7]. Such tissue-level interactions are mediated by both cytokines and matrix macromolecules. A considerable understanding has been gained regarding the molecular mechanisms by which cytokines affect cell behaviour. In general terms, these involve the following: cytokine ligation by specific cell surface receptors; receptor activation and initiation of a chain of intracellular phosphorylation/dephosphorylation events within diverse signalling networks; and resultant changes in the pattern of gene expression and/or functional state of the cytoskeleton. The inappropriate expression of cytokines and/or their receptors has long been recognized to contribute to the pathogenesis of breast cancer $[8,9]$.

In contrast to the well-documented involvement of cytokines in cancer development, matrix macromolecules have, until relatively recently, been considered to fulfil an exclusively structural role that is restricted to providing a scaffold for cell adhesion and migration. It is now clear, however, that common matrix constituents elicit signal transduction cascades as a result of their ligation by integrin receptors, and that such matrix-induced signalling networks share many common pathways with their cytokine-induced counterparts [10].

An important biological concept that has emerged during the past decade is that the bioactivity of cytokines and matrix macromolecules are mutually interdependent, in the sense that cytokines and matrix macromolecules exert reciprocal effects on their respective syntheses (i.e. cytokines affect the expression of matrix molecules, matrix receptors and matrix-degrading enzymes, whereas matrix macromolecules modulate the synthesis of cytokines and their respective cell surface receptors) [11]. In addition, the effects of cytokines on cell behaviour may be mediated by the matrix molecules whose synthesis they regulate (i.e. the observed cellular response to a cytokine may be a secondary consequence of the bioactivity of the matrix molecules whose expression is under primary cytokine control) [11]. Also, matrix molecules bind cytokines and present them to cell surface receptors in a functionally optimal state [12]. Finally, matrix macromolecules modulate cellular response to cytokines (i.e. the nature of the macromolecular matrix to which the cells are adherent determines cellular response to cytokines) $[11,13]$.

With respect to the latter findings, we reported that the nature of the macromolecular substratum critically determines whether transforming growth factor- $\beta_{1}$ stimulates or inhibits cell migration and hyaluronan synthesis by dermal fibroblasts [14]. Taken in conjunction with other published reports [15], these observations provide the basis of the 'tissue response unit' hypothesis [16]. That hypothesis states that the precise effect of a multifunctional cytokine on cell behaviour is determined by several tissue-level parameters, including the presence of other cytokines, the nature of the macromolecular matrix and the state of target cell activation. A clear corollary of this model is that the documented presence of a cytokine during the course of tumour progression is not sufficient in itself to provide an insight into its functional significance, in the absence of complementary data regarding alterations in stroma composition and/or physical organization caused by concurrent, pathology-associated matrix remodelling.

In addition to the above mechanisms, cell behaviour is also modulated by the topology of cell-matrix contact. For example, we have previously reported that vascular endothelial cells attached to the two-dimensional surface of a native type I collagen gel continue to express a resting ('cobblestone') phenotype for many weeks when cultured in the absence of exogenous angiogenic factors $[17,18]$. We also demonstrated that these cells adopt an angiogenic (sprouting) phenotype within $24 \mathrm{~h}$ of replating within the three-dimensional gel matrix, again in the complete absence of exogenous angiogenic factors $[17,18]$. In this situation, the only alteration is a shift from conditions in which the cells contact the collagenous matrix solely along their basal surface (and can therefore establish an apicalbasal polarity) to culture within an isotropic environment, in which contact with the matrix is made at sites that are distributed uniformly along the entire plasma membrane. Under these conditions, the observed changes in cell phenotype, which distinguish resting and angiogenic cells, are likely to be mediated by topology-dependent alterations in cytoskeletal organization and functionality [19]. 


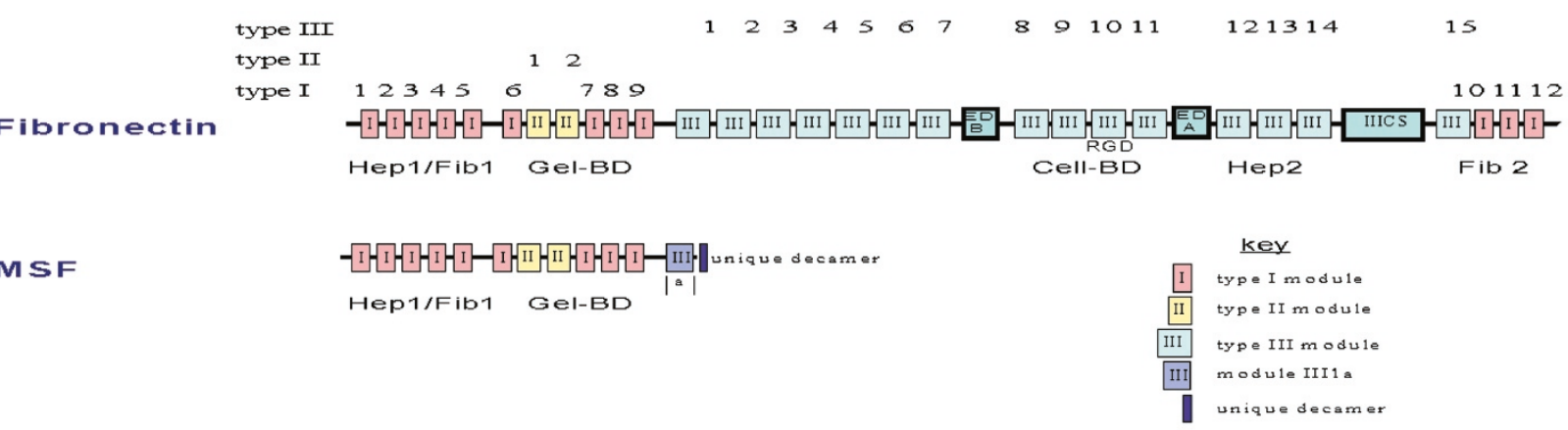

Structural homology between the amino-terminus of fibronectin and migration stimulating factor (MSF). MSF is identical to the 70 kDa aminoterminus of fibronectin, with the addition of a novel 10 amino sequence at its carboxyl-terminus.

Extrapolating to the in vivo situation, we suggest that the movement of resting endothelial cells from their twodimensional luminal position into the three-dimensional stromal compartment may be sufficient to induce changes in gene expression that are of relevance to tumourinduced angiogenesis. According to this view, angiogenic factors produced by the tumour may principally function to induce the initial stages of endothelial cell activation and migration into the stroma, and to provide a chemotactic stimulus for the subsequent directed migration of the resultant sprouting cells toward the tumour.

\section{Matrikines}

An upregulation in the expression of various classes of proteinase has long been recognized to be associated with tumour progression. Certain proteinases (e.g. stromelysin-3) may be described as oncofoetal in that they are constitutively expressed by foetal cells and reexpressed by activated adult cells during the course of tumour progression [20]. A combination of immunolocalization and in situ hybridization studies indicated that these matrix degrading enzymes are commonly coexpressed by both carcinoma and activated stromal cells [21], and available data suggest that the observed anatomical pattern of expression results from reciprocal tumour-stroma interactions [22].

Tumour-associated proteinases have been suggested to contribute to disease progression by a number of means, the most commonly discussed being degradation of basement membrane (thereby facilitating local invasion and metastasis) and release of matrix-bound cytokines [23]. Proteinases may also contribute to cancer progression by degrading matrix molecules into discrete peptide fragments, which display potent bioactivities that are not expressed (i.e. cryptic) by the full-length molecule [23-25]. The ubiquitous matrix macromolecule fibronectin and its proteolytically generated peptide fragments have been particularly well characterized in this regard [26].
Fibronectin is a modular glycoprotein (molecular mass approximately $250 \mathrm{kDa}$ ) that consists of a number of protease-resistant functional domains, so named on the basis of their specific binding affinity for other matrix macromolecules and members of the integrin family of matrix receptors (Fig. 1). These domains are as follows: Hep1/Fib1 (low affinity binding to heparin and fibrin), Gel-BD (binding to gelatin/collagen), Cell-BD (binding to integrin receptors on cell surface), Hep2 (high affinity heparin binding) and Fib2 (second fibrin binding site). Each functional domain is in turn composed of three possible homology modules (types I, II and III), which contain approximately 45, 60 and 90 amino acids, respectively. Considerably smaller amino acid recognition sequences (such as the RGD tripeptide) function as the minimal peptide motif required for ligation by integrin receptors [27]. All proteolytically generated functional domains of fibronectin, with the notable exception of the $43 \mathrm{kDa}$ gelatin-binding domain (Gel-BD), have been reported to express a diverse range of bioactivities, including effects on cell migration, adhesion, proteinase expression and differentiation [26].

The motogenic activity of full-length fibronectin and its proteolytically generated functional domains have most commonly been studied in variations of the transmembrane (or 'Boyden chamber') assay. Previous studies using this assay (for review [28]) have indicated that concentrations of fibronectin and its cell-binding domain in the region of $1-100 \mu \mathrm{g} / \mathrm{ml}$ stimulate the migration of human skin fibroblasts, as well as various other normal and transformed cell types. Ligation of the RGD amino acid motif (which is present in module III-10) by integrin receptors (e.g. $\alpha_{5} \beta_{1}$ ) is responsible for mediating motogenic activity.

In view of the important role played by the matrix in modulating cellular response to soluble effector molecules, we developed an alternative migration assay that involves cell attachment and movement within a three-dimensional matrix of native type I collagen fibres. Using this assay, we 
reported that purified gelatin-binding domain (Gel-BD) stimulates the migration of human skin fibroblasts, exhibiting a bell-shaped dose-response curve with half-maximal activity manifest at $0.1-1.0 \mathrm{pg} / \mathrm{ml}$ (i.e. approximately 2-20 pmol/l) [28]. This unexpected and unprecedented potent motogenic response was further shown to be substratum dependent (i.e. manifested by fibroblasts adherent to a native, but not denatured, type I collagen substratum). The strictly substratum-dependent nature of Gel-BD motogenic activity provides an explanation for the marked discrepancy between results obtained in the collagen gel assay (employing a native collagen substratum) and the majority of published studies using the transmembrane assay (employing denatured collagen-coated membranes).

Subsequent studies indicated that synthetic peptides containing the IGD tripeptide motif (as present with modules I7 and 19 of Gel-BD) essentially mimicked the motogenic activity of Gel-BD, including its substratum dependence [29]. Manifestation of IGD motogenic activity was temporally associated with an increase in the tyrosine phosphorylation of focal adhesion kinase (FAK125) and was inhibited by integrin $\alpha_{v} \beta_{3}$ neutralizing antibody [29].

On the basis of these various observations, we suggest the generic term 'matrikine' to define proteolytically generated fragments of matrix macromolecules, which display cryptic, cytokine-like bioactivities that are not expressed by their full length precursors. Taken together, these observations reveal a novel functionality of locally produced proteinases and matrix remodelling during tumour progression and other pathologies. They also indicate that, in addition to its other effects on cell behaviour, the extracellular matrix may act as a reservoir of soluble multifunctional bioregulatory molecules.

\section{Migration stimulating factor}

We previously reported that foetal skin fibroblasts migrate into gels of native type I collagen to a significantly greater extent than do corresponding adult cells [30]. We also reported that fibroblasts obtained from approximately $50 \%$ of sporadic breast cancer patients and more than $90 \%$ of patients with familial disease also express a foetal-like migratory phenotype [31-33]. In marked contrast, only $10-15 \%$ of fibroblasts obtained from age-matched and sex-matched healthy control individuals in the above studies exhibited a foetal-like pattern of migration. Studies concerned with the biochemical basis of these observations indicated that both foetal fibroblasts and the foetallike fibroblasts from cancer patients secrete a soluble MSF that is not produced by their normal adult counterparts [34]. Subsequent studies revealed that detectable quantities of MSF were present in the serum of more than $90 \%$ of breast cancer patients, compared with only 10-15\% age-matched and sex-matched control individuals [35]. Initial biochemical characterization of MSF purified from foetal and cancer patient fibroblast conditioned medium indicated that it had a molecular mass of approximately $70 \mathrm{kDa}$ and contained a peptide sequence identical to a region of the gelatin-binding domain of fibronectin $[36,37]$.

MSF has been cloned from a foetal fibroblast cDNA library (EMBL accession number AJ276395; Schor SL et al., manuscript submitted). Complete sequence data indicate that it is identical to the $5^{\prime}$ end of fibronectin cDNA (including a $56 \mathrm{bp}$ untranslated sequence), with the addition of a novel $175 \mathrm{bp} 3^{\prime}$ tail that consists of a $30 \mathrm{bp}$ coding sequence, followed by an untranslated region that contains five in-frame stop codons and a cleavage/polyadenylation signal.

Chromosome mapping and polymerase chain reaction data using template genomic DNA indicate that MSF is a truncated isoform of fibronectin, and that its specific $175 \mathrm{bp} 3^{\prime}$ tail is generated by retention of the intron separating fibronectin exons III $1 \mathrm{a}$ and III $\mathrm{b}$ and its cleavage during subsequent mRNA maturation. The deduced MSF protein consists of the intact $70 \mathrm{kDa}$ amino-terminus of fibronectin (containing the entire Fib1/Hep1 and Gel-BD regions, and the first portion of module III1); and a unique 10 amino acid carboxyl-terminus (VSIPPRNLGY), as coded by the first 30 $\mathrm{bp}$ of the retained intron (Fig. 1). All previously identified fibronectin isoforms (of which there are approximately 20) are 'full-length' compared with MSF and are produced by well characterized alternative splicing events at three sites downstream from the newly identified MSF splice site (i.e. the exons coding for the ED-A and ED-B type III homology modules, as well as the IIICS region; Fig. 1).

Recombinantly expressed human MSF stimulates the migration of adult skin fibroblasts with a dose-response relationship that is identical to that of Gel-BD [25] (Schor SL et al., unpublished data). Significantly, MSF motogenic activity is completely abolished by an anti-Gel-BD monoclonal antibody and is unaffected by antibodies raised against all other fibronectin functional domains, including the amino-terminal Fib1/Hep1 domain. Taken together, these (and related observations) suggest that MSF motogenic activity is mediated by a peptide region that is located within its gelatin-binding domain; and that the IGD motif is a prime candidate bioactive sequence. As is the case with Gel-BD, fibroblast motogenic response to recombinant human MSF is strictly matrix dependent, being manifest by cells that are adherent to native, but not denatured, type I collagen. Recombinant human MSF and GelBD both express a number of other relevant bioactivities, including the stimulation of hyaluronic acid synthesis [38] and the induction of a potent angiogenic response in the chick yolk sac membrane (Schor AM, unpublished data).

On the basis of these various observations, we conclude that MSF and Gel-BD are functionally equivalent, but pro- 




The expression and functionality of migration stimulating factor (MSF) during the progression of breast cancer. MSF is concurrently expressed by a proportion of carcinoma cells, stromal fibroblasts and vascular endothelial cells (producer cells). In vitro and in vivo observations indicate that it affects a diverse range of phenotypic features of these same cells (now functioning as target cells). MSF activity may be mediated by autocrine mechanims (i.e. same producer and target cell population) and paracrine mechanisms (i.e. different producer and target cell populations). Both MSF expression and cellular response to it are matrix dependent. HA, hyaluronic acid.

duced by distinct mechanisms, presumably under independent control (i.e. Gel-BD is a matrikine that is generated by the proteolytic degradation of matrix fibronectin, whereas MSF is the first genetically truncated isoform of fibronectin identified). It should also be noted that MSF differs from all fragments of full-length fibronectin isoforms (including Gel-BD) in terms of its unique 10 amino acid carboxyl-terminus. The generation of MSF by a genetic mechanism therefore affords the cell with a means to selectively produce a functional equivalent to Gel-BD without the necessity of producing a mixture of proteinase generated matrix fragments that express a complex array of cryptic bioactivities.

Using an MSF-specific antisense ribroprobe (to a sequence contained within its unique $175 \mathrm{bp} 3^{\prime}$ tail), in situ hybridization revealed that MSF is weakly expressed by a small number of ductal epithelial cells and interlobular fibroblasts in normal breast, and is more strongly expressed by carcinoma cells, stromal fibroblasts and some blood vessels in breast tumours (Schor SL, unpublished data). These observations are consistent with data from our laboratory that indicate that MSF is constitutively expressed by interlobular, but not intralobular fibroblasts isolated from normal breast tissue [39]. Thus, they indicate for the first time that MSF is abundantly and concurrently expressed by breast carcinoma cells, as well as tumour-associated fibroblasts and vascular endothelial cells. Complementary immunolocalization using an MSF-specific monoclonal antibody confirmed the association of MSF protein with both mammary carcinoma and stromal cells. The expression of MSF by both carcinoma and tumour-associated stromal cells is consistent with published data that indicate a similar concurrent expression of full-length oncofoetal fibronectin isoforms by these cells [40]. A functional co-operation between MSF and previously described oncofoetal fibronectins in the induction of new blood vessels is suggested by the angiogenic activity of MSF and the reported expression of oncofoetal fibronectins that contain the ED-A and ED-B domains in vascular-rich stroma $[41,42]$.

It should finally be noted that the presence of MSF in tumour tissues (as indicated by both immunolocalization and in situ hybridization) is not in itself sufficient to conclude that MSF bioactivity is expressed. In accord with the tissue response unit hypothesis, the effect of modulating parameters, such as the nature of the extracellular matrix and cytokine profile, must also be taken into account. With respect to the latter, we previously reported that transforming growth factor- $\beta_{1}$ is a potent inhibitor of MSF and Gel-BD functionality $[28,43]$. Our current understanding of MSF expression and functionality during breast cancer progression is summarized in Fig. 2.

\section{Origins of stromal cell phenotypic diversity}

We originally speculated that 'foetal-like' fibroblasts were associated with breast and other types of cancer, and that the resultant perturbations in signalling between stroma and epithelium played an important role in driving the process of tumour progression [4,5]. In these models, fibroblasts were defined as 'foetal-like' on the basis of their re-expression of molecules (such as MSF) that are constitutively expressed during development. Subsequent studies considerably extended these analyses to include many other gene products $[44,45]$ and explicitly went on to support the view that stromal 'foetalization' is an important feature of carcinogenesis [46].

These variations in stromal cell phenotype may arise through both genetic and epigenetic mechanisms. With 
respect to the former, Moinfar et al. [47] recently presented evidence documenting loss of heterozygosity in DNA isolated from microdissected regions of mammary tumour stroma that were distinct from those associated with the carcinoma cells. These observations support the view that environmental carcinogens that were previously implicated in the development of breast and other cancers also produce genetic lesions in stromal cells, and that this genetic damage may also contribute to the course of disease progression. Alterations in stromal cell phenotype may additionally arise from epigenetic mechanisms that involve responses to altered signalling from a progressively aberrant epithelium and to stress-inducing microenvironmental agents (as contained in cigarette smoke). In this context, we recently observed that a number of cytokines and genotoxic agents 'switch on' MSF expression by adult skin fibroblasts and that, under certain circumstances, this alteration in gene expression is persistent for many months in vitro (Schor SL et al., unpublished data).

\section{Conclusion}

An important function of stromal cells is the deposition and remodelling of the extracellular matrix. In addition to providing the physical basis for tissue integrity, it is now clear that matrix macromolecules exert profound effects on cell behaviour by a variety of mechanisms. These include the following: receptor-mediated signal transduction; modulation of cellular response to cytokines; binding and presenting cytokines in an optimal conformation; and acting as a reservoir for proteinase-generated matrikines. Truncated isoforms of matrix molecules produced by alternative splicing (such as MSF) may also express a range of bioactivities that are cryptic within the full-length molecule. The substratum dependence of Gel-BD and MSF serve to emphasize the critical importance of the physical organization of the matrix in terms of its effect on the behaviour of adherent cells.

We previously speculated that expression of MSF and other oncofoetal effector molecules by the foetal-like (activated) stromal cells in cancer patients may contribute to disease progression by creating a milieu that promotes the clonal expansion and manifestation of invasive behaviour of the emerging (pre)malignant cell population [5]. In this regard, it is important to bear in mind that tumour progression is a relatively indolent process, in which many decades may elapse between inception of the initiating genetic lesion and the emergence of a clinically recognizable malignancy. Available data suggest that the proportion of 'initiated' cells that eventually develop into a tumour is quite low. We accordingly suggest that factors that may alter the kinetics of progression may play an important, and perhaps decisive, role in determining the probability of disease inception. It is in this postulated role of an 'accelerator' of cancer progression that we envision the contribution of activated 'foetal-like' cells (and the MSF they produce) to cancer pathogenesis. Seen in this light, MSF functions as a severity (rather that causative) agent. The documented bioactivities of MSF (motogenic, angiogenic and stimulation of hyaluronan synthesis) are all potentially relevant features of its functionality. In the light of these findings, we suggest that the development of adjunct therapies that are specifically designed to normalize activated cell function may prove clinically efficacious in the treatment of patients with cancer and/or the retardation of cancer development in healthy individuals who are deemed to be at elevated risk.

\section{Acknowledgements}

Work summarized in the present review was done in collaboration with Drs R Baillie, J Banyard, J Clausen, I Ellis, R Kay and K Seneveratne. Mrs J Cox is gratefully acknowledged for her excellent technical support. The authors also wish to thank Professor A Howell and Mr G Rushton (CRC Department of Medical Oncology, Christie Hospital, Manchester) for their invaluable support and contribution to the initial identification and characterization of MSF. Studies leading to the observations reported within this manuscript were funded by the Cancer Research Campaign, Medical Research Council and Tayside Area Oncology Fund.

\section{References}

1. Beckmann MW, Niederacher D, Schnurch HG, Gusterson BA, Bemnder HG: Multistep carcinogenesis of breast cancer and tumour heterogeneity. J Mol Med 1997, 75:429-439.

2. Ilyas M, Straub J, Tomlinson IP, Bodmer WF: Genetic pathways in colorectal and other cancers. Eur J Cancer 1999, 35:1986-2002.

3. Sager R: Expression genetics in cancer: shifting the focus from DNA to RNA. Proc Natl Acad Sci USA 1997, 94:952-955.

4. Schor SL, Schor AM, Howell A, Crowther D: Hypothesis: persistent expression of fetal phenotypic characteristics by fibroblasts is associated with an increased susceptibility to neoplastic disease. Exp Cell Biol 1987, 55:11-17.

5. Schor SL, Schor AM: Stromal acceleration of tumour progression: role of 'fetal-like' fibroblast subpopulations. Pathol Update 1997, 4:75-95.

6. Noel A, Foidart JM: The role of the stroma in breast carcinoma growth in vivo. J Mammary Gland Biol Neoplasia 1998, 3:215225.

7. Mackenzie I: Epithelial-mesenchymal interactions in the development and maintenance of epithelial tissues. In: The Keratinocyte Handbook. Edited by Leigh IM, Lane EB, Watt FM. Cambridge: Cambridge University Press, 1994:243-258.

8. de Jong JS, van Diest PJ, van der Valk P, Baak JP: Expression of growth factors, growth inhibitory factors and their receptors in invasive breast cancer: an inventory in search of autocrine and paracrine loops. J Pathol 1998, 184:44-52.

9. Rasmussen AA, Cullen: Paracrine/autocrine regulation of breast cancer by insulin-like growth factors. Breast Cancer Res Treat 1998, 47:219-233.

10. Juliano R: Cooperation between soluble factors and integrinmediated cell anchorage in the control of cell growth and differentiation. Bioessay 1996, 18:911-917.

11. Schor SL: Cytokine control of cell motility: modulation and mediation by the extracellular matrix. Prog Growth Factor Res 1994, 5:223-248.

12. Streuli C: Extracellular matrix remodelling and cellular differentiation. Curr Opin Cell Biol 1999, 11:634-640.

13. Lee YJ, Strueli CH: Extracellular matrix selectively modulates the response of mammary epithelial cells to different soluble signaling ligands. J Biol Chem 1999, 274:22401-22408.

14. Ellis I, Schor SL: The interdependent modulation of hyaluronan synthesis by TGF- $\beta 1$ and extracellular matrix: consequences for the control of cell migration. Growth Factors 1995, 12:211222.

15. Madri JA, Pratt BM, Tucker AM: Phenotypic modulation of endothelial cells by transforming growth factor-beta depends upon the composition and organization of the extracellular matrix. J Cell Biol 1988, 106:1375-1384. 
16. Ellis IR, Banyard J, Schor SL: Motogenic and biosynthetic response of adult skin fibroblasts to TGF isoforms $(-1,-2$, and -3) determined by 'tissue response unit': role of cell density and substratum. Cell Biol Int 1999, 23:593-602.

17. Schor AM, Schor SL, Allen TD: Effects of culture conditions on the proliferation and morphology of bovine aortic endothelial cells in vitro: reversible expression of the sprouting cell phenotype. J Cell Sci 1983, 62:267-285.

18. Canfield AE, Boot-Handford RP, Schor AM: Thrombospondin gene expression by endothelial cells in culture is modulated by cell proliferation, cell shape and the substratum. Biochem J 1990, 268:225-230.

19. Ingber DE: Tensegrity: the architectural basis of cellular mechanotransduction. Annu Rev Physiol 1997, 59:575-599.

20. Bassett P, Bellocq JP, Wolf C, Stoll I, Hutin P, Limacher JM, Podhajcer OL, Chenard MP, Rio MC, Chambon P: A novel metalloproteinase gene specifically expressed in stromal cells of breast carcinomas. Nature 1990, 348:699-704.

21. Lebeau A, Nerlich AG, Sauer U, Lichtinghagen R, Lohrs U: Tissue distribution of major matrix metalloproteinases and their transcripts in human breast carcinomas. Anticancer Res 1999, 19:4257-4264.

22. Heppner KJ, Matrisian LM, Jensen RA, Rodgers WH: Expression of most matrix metalloproteinase family members in breast cancer represents a tumor-induced host response. $A m \mathrm{~J}$ Pathol 1996, 149:273-282.

23. McCawley LJ, Matrisian LM: Matrix metalloproteinases: multifunctional contributors to tumor progression. Mol Med Today 2000, 6:149-156.

24. Davis GE, Bayless KJ, Davis MJ, Meininger GA: Regulation of tissue injury responses by exposure of matricryptic sites within extracellular matrix molecules. Am J Pathol 2000, 156: 1489-1498.

25. Stetler-Stevenson WG, Yu AE: Proteases in invasion: matrix metalloproteinases. Semin Cancer Bio/ 2001, 11:143-153.

26. Fukai F, Ohtaki M, Fugii N, Yajima H, Ishii T, Nishizawa Y, Miyazaki $\mathrm{K}$, Katayama T: Release of biological activities from quiescent fibronectin by a conformational change and limited proteolysis by matrix metalloproteinases. Biochemistry 1995, 34: 11453-11459.

27. Newham $\mathrm{P}$, Humphries MJ: Integrin adhesion receptors: structure, function and implications for biomedicine. Mol Med Today 1996, 2:304-313.

28. Schor SL, Ellis I, Dolman C, Banyard J, Humphries MJ, Mosher DF, Grey AM, Mould AP, Sotille J, Schor AM: Substratumdependent stimulation of fibroblast migration by the gelatinbinding domain of fibronectin. J Cell Sci 1996, 109: 2581-2590.

29. Schor SL, Ellis I, Banyard J, Schor AM: Motogenic activity of the IGD amino acid motif. J Cell Sci 1999, 112:3879-3888.

30. Schor SL, Schor AM, Rushton G, Smith L: Adult, fetal and transformed fibroblasts display different migratory phenotypes on collagen gels: evidence for an isoformic transition during fetal development. J Cell Sci 1985, 73:221-234.

31. Durning P, Schor SL, Sellwood RA: Fibroblasts from patients with breast cancer show abnormal migratory behaviour in vitro. Lancet 1984, 2:890-892.

32. Schor SL, Haggie JA, Durning $P$, Howell A, Sellwood RA, Crowther D: Occurrence of a foetal fibroblast phenotype in familial breast cancer. Int J Cancer 1986, 37:831-836.

33. Haggie J, Schor SL, Howell A, Birch JM, Sellwood RAS: Fibroblasts from relatives of hereditary breast cancer patients display fetal-like behaviour in vitro. Lancet 1987, 11455-1457.

34. Schor SL, Schor AM, Grey AM, Rushton G: Foetal and cancer patient fibroblasts produce an autocrine migration stimulating factor not made by normal adult cells. J Cell Sci 1988, 90:391. 399.

35. Picardo M, Schor SL, Grey AM, Howell A, Laidlaw I, Redford J, Schor AM: Migration stimulating activity in the serum of breast cancer patients. Lancet 1991, 337:130-133.

36. Grey AM, Schor AM, Rushton G, Ellis I, Schor SL: Purification of the migration stimulating factor produced by fetal and cancer patient fibroblasts. Proc Natl Acad Sci USA 1989, 86:24382442.

37. Schor SL, Grey AM, Ellis I, Schor AM, Coles B, Murphy R: Migration stimulating factor: its structural homology to the gelatinbinding domain of fibronectin, mode of action and possible function in health and disease. In: Cell Behaviour: Adhesion and Motility, S.E.B. Symposium No. 47. Edited by Evans G, Wigley C, Warn R. 1993:235-251.

38. Schor SL, Schor AM, Grey AM, Chen J, Rushton G, Grant ME, Ellis I: Mechanism of action of the migration stimulating factor (MSF) produced by fetal and cancer patient fibroblasts: effect on hyaluronic acid synthesis. In Vitro Cell Dev Biol 1989, 25: 737-746.

39. Schor AM, Rushton G, Ferguson JE, Howell A, Redford J, Schor SL: Phenotypic heterogeneity in breast fibroblasts: functional anomaly in fibroblasts from histologically normal tissue adjacent to carcinoma. Int J Cancer 1994, 59:25-32.

40. Midulla M, Verma R, Pignatelli M, Ritter MA, Courtenay-Luck NS, George AJ: Source of oncofetal ED-B fibronectin: implications of production by both tumor and endothelial cells. Cancer Res 2000, 60:164-169.

41. Castellani P, Viale G, Dorcaratto A, Nicolo G, Kaczmarek J, Querze G, Zarde L: The fibronectin isoform containing the EDB oncofetal domain: a marker of angiogenesis. Int J Cancer 1994, 59:612-618.

42. Brown LF, Guidi AJ, Schnitt SJ, Van De Water L, Iruela-Arispe ML, Yeo TK, Tognazzi K, Dvorak HF: Vascular stroma formation in carcinoma in situ, invasive carcinoma and metastatic carcinoma of the breast. Clin Cancer Res 1999, 5:1041-1056.

43. Ellis I, Grey AM, Schor AM, Schor SL: Antagonistic effects of transforming growth factor beta and MSF on fibroblast migration and hyaluronic acid synthesis: possible implications for wound healing. J Cell Sci 1992, 102:447-456.

44. Spanakis E, Brouty-Boye D: Quantitative variation in protooncogene and cytokine gene expression in isolated breast fibroblasts. Int J Cancer 1995, 61:698-705.

45. Spanakis E, Brouty-Boye D: Discrimination of fibroblast subtypes by multivariate analysis of gene expression. Int $J$ Cancer 1997, 71:402-409.

46. Singer CF, Rasmussen A, Lippman ME, Cullen KJ: Coexpression of stromelysin-3 and insulin-like growth factor II in tumors of ectodermal, mesodermal and endodermal origin: indicator of a fetal cell phenotype. J Clin Endocrinol Metab 1997, 82:19171922.

47. Moinifar F, Man YG, Arnould L, Bratthauer GL, Ratschek M, Tavassoli FA: Concurrent and independent genetic alterations in the stromal and epithelial cells of mammary carcinoma: implications for tumorigenesis. Cancer Res 2000, 60:25622566. 